\title{
CTF18 interacts with replication protein A in response to replication stress
}

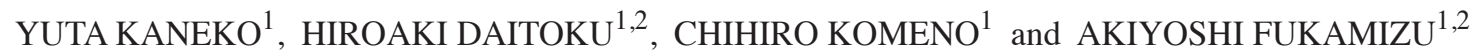 \\ ${ }^{1}$ Graduate School of Life and Environmental Sciences; ${ }^{2}$ Life Science Center, \\ Tsukuba Advanced Research Alliance, University of Tsukuba, Tsukuba, Ibaraki 305-8577, Japan
}

Received December 5, 2015; Accepted May 3, 2016

DOI: $10.3892 / \mathrm{mmr} .2016 .5262$

\begin{abstract}
Replication stress response is a protective mechanism against defects in chromosome replication for maintaining genome integrity in eukaryotic cells. An alternative clamp loader complex termed chromosome transmission fidelity protein 18 and replication factor C (CTF18-RFC) has been shown to act as a positive regulator of two types of replication stress response: S-phase checkpoint signaling and translesion DNA synthesis. However, it remains largely unknown how CTF18-RFC responds to replication stress and is recruited to stalled replication forks. The present study demonstrated that endogenous CTF18 forms a physical complex with a single-stranded DNA-binding protein replication protein A (RPA) in mammalian cells. Using an in situ proximity ligation assay (PLA), it was demonstrated that the interaction between CTF18 and RPA occurs in chromatin when replication stress is elicited by treatment with hydroxyurea during $\mathrm{S}$ phase. Similar results were obtained after exposure to ultraviolet irradiation, which triggers translesion DNA synthesis. Furthermore, the PLA demonstrated that the kinetics of the interaction between CTF18 and RPA was positively correlated with that of checkpoint kinase 1 phosphorylation, which is an indicator of activation of the ATM and Rad3-related pathway. These findings provide novel insights into the molecular mechanism underlying the participation of CTF18-RFC in the regulation of replication stress response.
\end{abstract}

\section{Introduction}

Chromosome replication is a risky process for maintaining genome integrity, as unrepaired DNA lesions at S phase interfere with the progress of replication forks and thereby result

Correspondence to: Professor Akiyoshi Fukamizu, Life Science Center, Tsukuba Advanced Research Alliance, University of Tsukuba, 1-1-1 Tennoudai, Tsukuba, Ibaraki 305-8577, Japan E-mail: akif@tara.tsukuba.ac.jp

Key words: chromosome transmission fidelity protein 18 , replication protein A, replication stress, S-phase checkpoint response, translesion DNA synthesis, in situ proximity ligation assay in excessive formation of single-strand DNA (ssDNA) that could be a major cause of deleterious lesions, such as DNA double-strand breaks. To preserve genome integrity during chromosome replication, eukaryotic cells have acquired several adaptive responses to DNA damage $(1,2)$. One of the most studied pathways is the S-phase checkpoint response, which is evoked by an exposed ssDNA at stalled replication forks, attributed to a deficiency in DNA synthesis. The checkpoint kinase ATM and Rad3-related (ATR) is recruited on ssDNA where it is coated with ssDNA-binding protein, replication protein A (RPA). It then causes the phosphorylation and activation of downstream checkpoint kinase 1 (Chk1), which in turn stabilizes replication forks for genome integrity (3-5). Another is a damage tolerance mechanism termed translesion DNA synthesis (TLS), the major process with which cells replicate past the unrepaired DNA lesion during $S$ phase (6). When the normal replication machinery is blocked at ultraviolet (UV)-induced cyclobutane pyrimidine dimers (CPDs), the Y-family DNA polymerase Polh replaces the stalled replicative DNA polymerase. This is dependent upon monoubiquitination of the ring-shaped clamp protein, proliferating cell nuclear antigen (PCNA), by the E3 ubiquitin ligase RAD18. Monoubiquitinated PCNA has an increased affinity for Polh, thus aiding the recruitment of Polh to stalled replication forks and allowing accurate replicative bypass of CPDs by incorporating correct bases on the opposite strand $(7,8)$. Consequently, TLS overcomes UV-induced replication blocks, thereby preventing sustained activation of the S-phase checkpoint in response to excessive formation of ssDNA (9).

Accumulating evidence has shown that the S-phase checkpoint and TLS are activated by conserved clamp loader complex termed chromosome transmission fidelity protein 18 and replication factor C (CTF18-RFC) (10-13). CTF18-RFC is one of four 'heteropentameric RFC complexes' each of which contains a common small subunit comprising RFC2-4 together with a unique larger subunit, including either RFC1, Elg1, RAD17 or CTF18. RFC1-RFC is important in normal DNA replication as it loads the homotrimeric PCNA clamp around the junction of primers with template DNA at replication forks (14). Elg1-RFC is involved in the maintenance of genome stability $(15,16)$, while RAD17-RFC contributes to the activation of the DNA damage checkpoint by loading the heterotrimeric 9-1-1 checkpoint clamp at sites of damaged DNA (17). In addition, although CTF18-RFC was originally 
reported to be important in establishing sister chromatid cohesion (18), recent studies with budding yeast have shown that CTF18-RFC mediates activation of the S-phase checkpoint depending on the association with DNA polymerase $\varepsilon$ (10). By contrast, a biochemical study with an in vitro reconstitution system has demonstrated that CTF18-RFC binds to and stimulates the DNA synthetic activity of DNA polymerase $\eta$ (11). However, the molecular mechanisms underlying these alternative functions of CTF18-RFC remain largely unknown.

In the present study, it was demonstrated that RPA is a novel binding partner of CTF18 in mammalian cells. Among the heterotrimeric subunits, RPA1 and RPA2 are detected as a complex with CTF18. Notably, the DuoLink in situ proximity ligation assay (PLA) demonstrated the nuclear interaction between CTF18 and RPA in response to replication stresses induced by hydroxyurea (HU) treatment and UV irradiation. Furthermore, the kinetics of CTF18-RPA interaction were positively correlated with the sustained activation of the ATR-Chk1 pathway after UV irradiation. The present findings provide insight into the mechanism underlying the functional role of CTF18 in replication stress responses.

\section{Materials and methods}

Cell culture. HEK293 cells (RIKEN BioResource Center, Tsukuba, Japan) were kept at $37^{\circ} \mathrm{C}$ in a humidified $5 \% \mathrm{CO}_{2}$ atmosphere and cultured in Dulbecco's modified Eagle's medium (Nacalai Tesque, Inc., Kyoto, Japan) supplemented with $10 \%$ fetal bovine serum (Gibco; Thermo Fisher Scientific, Inc., Waltham, MA, USA) and 100 units penicillin/streptomycin (Sigma-Aldrich, St. Louis, MO, USA).

Antibodies. The following antibodies were used in the present study: Rabbit polyclonal anti-CTF18 (cat no. A301-883A; 1:1,000 for western blot analysis; 1:100 for PLA; Bethyl Laboratories, Inc., Montgomery, TX, USA); rabbit monoclonal anti-RPA1 (cat. no. ab79398; 1:3,000 for western blot analysis) and mouse monoclonal anti-RPA2 (cat. no. ab2175; 1:2,000 for western blot analysis; 1:300 for PLA) (Abcam, Cambridge, MA, USA); mouse monoclonal anti- $\alpha$ tubulin (cat. no. B-5-1-2; 1:3,000 for western blot analysis; Sigma-Aldrich); mouse monoclonal anti-Chk1 (cat. no. K0086-3; 1:2,000 for western blot analysis; Medical \& Biological Laboratories, Nagoya, Japan); and rabbit polyclonal anti-phospho-Chk1 at Ser345 (cat. no. 2341 1:1,000 for western blot analysis; Cell Signaling Technology, Danvers, MA, USA). Secondary antibodies were polyclonal HRP-conjugated sheep anti-mouse (cat. no. NA931V; 1:30,000) and donkey anti-rabbit (cat. no. NA934V; 1:30,000) obtained from GE Healthcare Life Sciences (Little Chalfont, UK).

Cell synchronization. Cell cycle synchronization was performed by the double thymidine block method as reported previously (19). Briefly, exponentially growing HEK293 cells were treated with $2 \mathrm{mM}$ thymidine (Nacalai Tesque, Inc.) for $16 \mathrm{~h}$, thymidine-free media for $10 \mathrm{~h}$, and $2 \mathrm{mM}$ thymidine for $18 \mathrm{~h}$ to arrest the cell cycle at the G1/S boundary. Then, cells were released by changing the medium and analyzed at various time intervals. Cell synchronization was conducted prior to the in situ proximity ligation assay and western

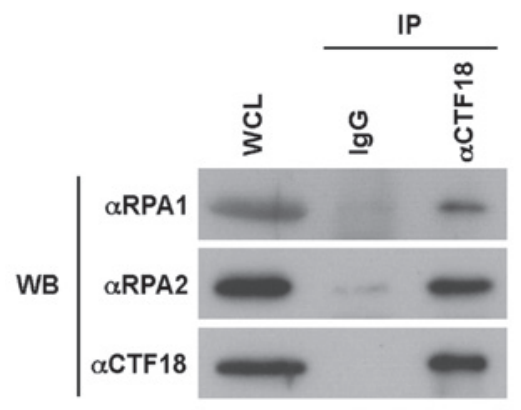

Figure 1. CTF18 forms a complex with RPA in HEK293 cells. Endogenous CTF18 interacts with RPA1 and RPA2. Whole-cell lysates from HEK293 cells were immunoprecipitated with normal IgG or anti-CTF18 antibody, followed by western blot analysis with antibodies as indicated. CTF18, chromosome transmission fidelity protein 18; RPA, replication protein A; IP, immunoprecipitation

blotting to detect phosphorylation of Chk1, however, the co-immunoprecipitation assay was performed with unsynchronized cells.

Co-immunoprecipitation assay and western blotting. Co-immunoprecipitation was performed as previously described (20). Briefly, whole-cell lysates were obtained from HEK293 cells using a lysis buffer [containing $20 \mathrm{mM}$ Hepes (pH 7.9), 150 mM NaCl, 0.1\% Triton X-100 ((Nacalai Tesque, Inc.) and protease inhibitor cocktail (Nacalai Tesque, Inc.)] were immunoprecipitated with normal IgG or anti-CTF18 antibody, and then separated by $10 \%$ SDS-PAGE. The proteins were transferred to a polyvinylidene difluoride membrane (EMD Millipore, Billerica, MA, USA) and blocked for $1 \mathrm{~h}$ with $0.3 \%$ skim milk at room temperature. The membrane was incubated overnight at $4^{\circ} \mathrm{C}$ with antibodies against RPA1, RPA2 and CTF18. Following washing with Tris-buffered saline with Tween 20 (TBST), the membrane was probed with the secondary antibodies for $1 \mathrm{~h}$ at room temperature and then washed again with TSBT. The membranes were visualized using the enhanced chemiluminescence system (Bio-Rad Laboratories, Inc., Hercules, CA, USA) and exposed to X-ray film (Fujifilm Corporation, Tokyo, Japan).

In situ proximity ligation assay (PLA). PLA was performed according to the manufacturer's instructions (Sigma-Aldrich). Briefly, synchronized HEK293 cells at S phase were treated with HU (0, 2 or $5 \mathrm{mM}$; Wako Pure Chemical Industries, Ltd., Osaka, Japan) or irradiated with UV light $(0,20$ or $100 \mathrm{~J} / \mathrm{m}^{2}$ ), after $2 \mathrm{~h}$, cells were extracted with CSK buffer (10 mM PIPES-NaOH, pH 6.8; 300 mM sucrose and $100 \mathrm{mM}$ $\mathrm{NaCl}$ ) containing $0.5 \%$ Triton $\mathrm{X}-100$ for $5 \mathrm{~min}$ for detection of chromatin bound proteins (21). After washing with CSK buffer without Triton X-100, cells were fixed with $3.7 \%$ formalin (Wako Pure Chemical Industries, Ltd.) for $20 \mathrm{~min}$, followed by permeabilization with ice-cold methanol for $10 \mathrm{~min}$. After blocking with Duolink Blocking solution (Sigma-Aldrich), cells were probed with mouse monoclonal anti-RPA2 and rabbit polyclonal anti-CTF18 antibodies, and then mouse or rabbit PLA probes were added. Hybridization of the oligonu- 

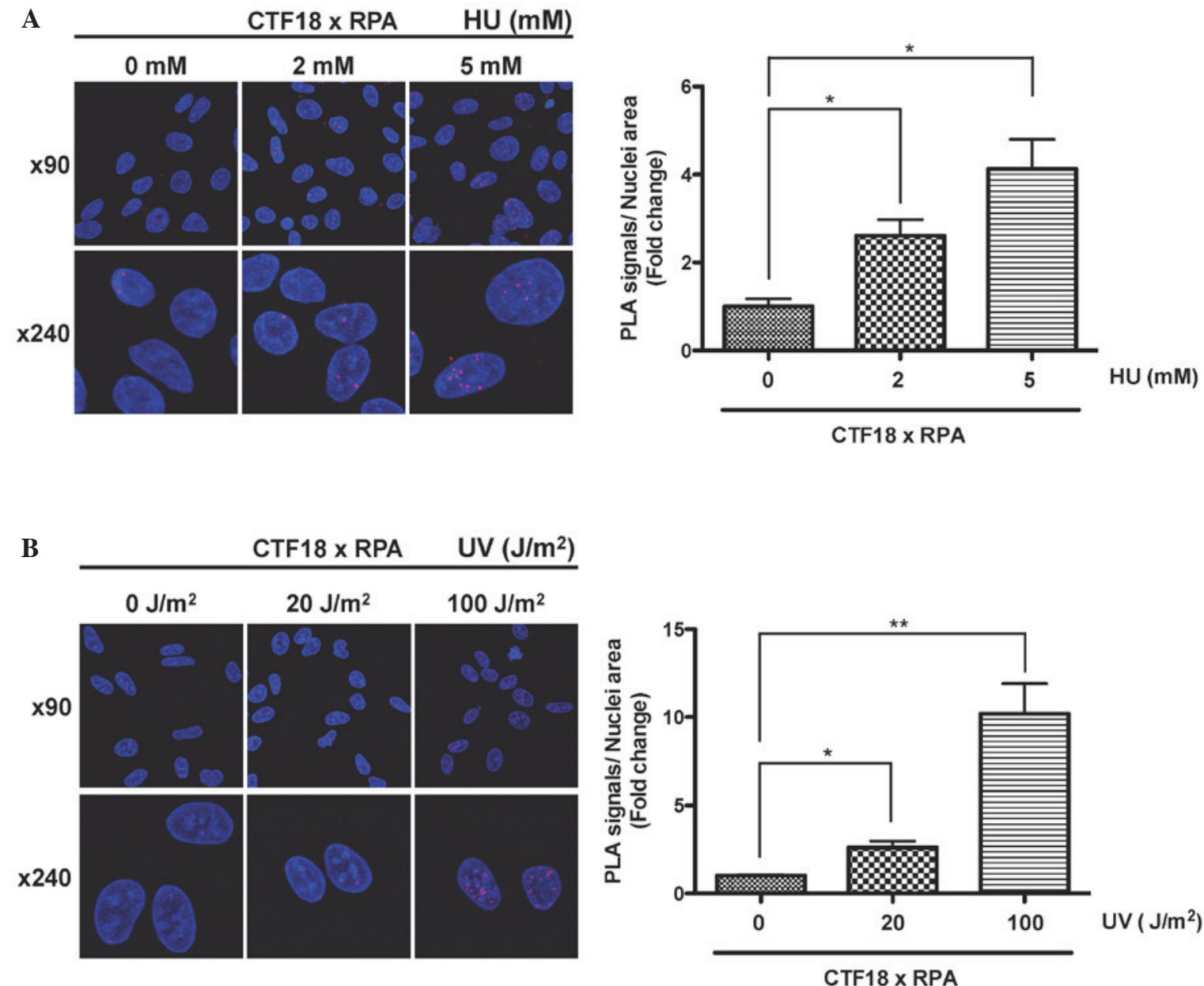

Figure 2. CTF18 binds to RPA in response to replication stresses. (A) The interaction between CTF18 and RPA occurs after treatment with HU. HEK293 cells were synchronized to S phase by double thymidine block and then treated with the indicated concentration of HU. After $2 \mathrm{~h}$, cells were treated with CSK/Triton-X buffer followed by fixation, and then an in situ PLA was performed with anti-RPA2 and anti-CTF18 antibodies. The red fluorescent foci indicate the proximity of the two proteins (magnification, $\mathrm{x} 90$ or x240). Duolink Image Tool was used to quantify PLA signals ( $\mathrm{n}=3$, x 90 magnification). The vertical axis shows the total nuclear PLA signals divided by nuclei area and normalized to non-treatment group, and the horizontal axis indicates the concentration of HU. Error bars indicate the standard error of the mean of three different fields. (B) The CTF18-RPA interaction occurs after exposure to UV irradiation. HEK293 cells were synchronized to S phase by double thymidine block and then irradiated with indicated dose of UV light. The PLA was performed as shown in (A). Nuclei were stained with Hoechst $33342 .{ }^{*} \mathrm{P}<0.05,{ }^{* *} \mathrm{P}<0.01$. CTF18, chromosome transmission fidelity protein 18; RPA, replication protein A; PLA, proximity ligation assay; HU, hydroxyurea; UV, ultraviolet.

cleotide arms of the PLA probes creates a template for rolling circle amplification (RCA) only when the epitopes of the target proteins are in close proximity $(<40 \mathrm{~nm})$. Following amplification of the RCA, an oligonucleotide probe labeled with Texas Red fluorophore is added and hybridizes with the RCA product. All fluorescence data were obtained with a confocal microscope FV10i (Olympus Corporation, Tokyo, Japan) and z-stacked images (collected in $1 \mu \mathrm{m}$ steps) were used for quantification of PLA signals with the Duolink Image Tool (version 1.0; Sigma-Aldrich). The PLA signals were calculated from three different fields and the scores were converted into fold change compared with the control.

Statistical analyses. All experiments were repeated in triplicate. Statistical significance for in situ PLA was determined by two-tailed unpaired Student's t-test using Graphpad Prism 5 (Graphpad Software, Inc., La Jolla, CA, USA). $\mathrm{P}<0.05$ was considered to indicate a statistically significant difference.

\section{Results}

CTF18 interacts with the RPA complex in HEK293 cells. To elucidate the mechanism underlying replication stress responses by CTF18-RFC, the present study aimed to identify a new binding partner for CTF18. It focused on a ssDNA-binding protein RPA that stabilizes the ssDNA region during DNA replication and repair, and also acts as a scaffold for DNA processing proteins. Although RPA is a heterotrimeric complex composed of 70, 32 and $14 \mathrm{kDa}$ subunits, referred to as RPA1, RPA2 and RPA3, respectively (22), the present study investigated the interaction between endogenous CTF18 with two RPA subunits, RPA1 and RPA2, which are detectable with specific antibodies. As shown in Fig. 1, an immune-complex of CTF18 from whole cell lysates of HEK293 cells included RPA1 and RPA2, suggesting that CTF18 forms a complex with RPA in vivo.

CTF18 is associated with RPA in response to replication stress. If the interaction between CTF18 and RPA occurs in the 


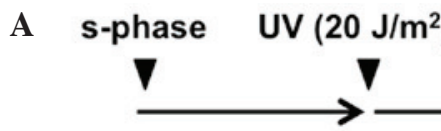

$\mathrm{Oh}$

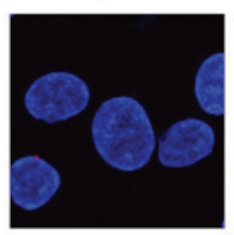

$2 \mathrm{~h}$

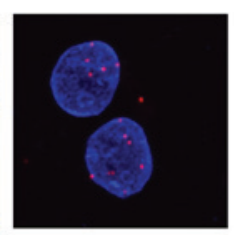

$4 \mathrm{~h}$

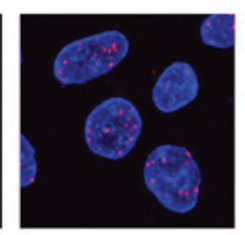

$6 \mathrm{~h}$

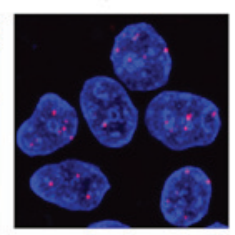

$8 \mathrm{~h}$

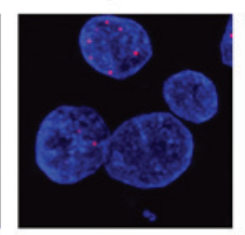

$10 \mathrm{~h}$

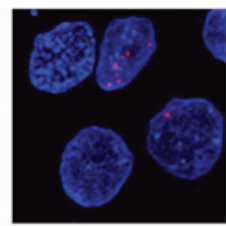

CTF18 x RPA / UV $\left(20 \mathrm{~J} / \mathrm{m}^{2}\right)$

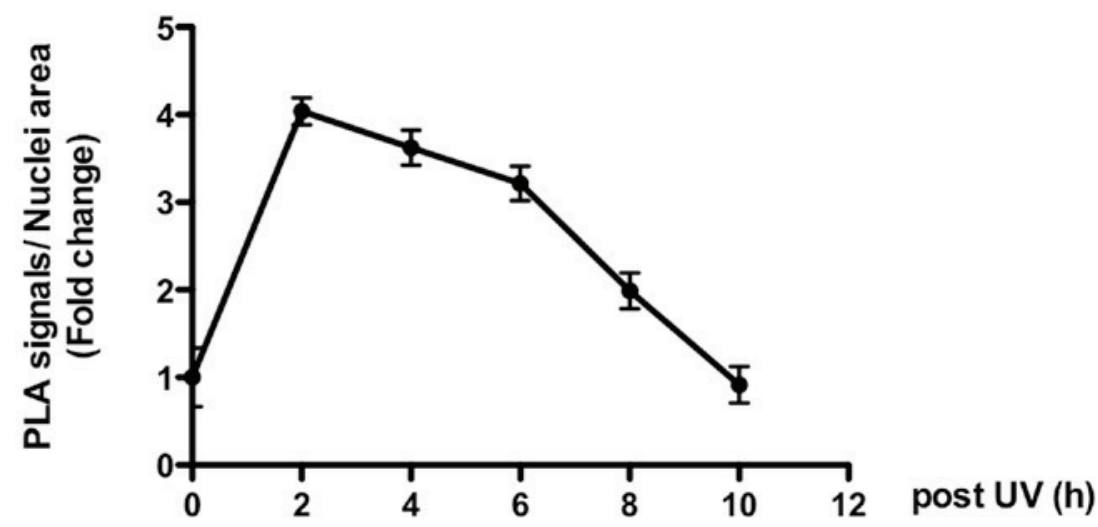

UV $\left(20 \mathrm{~J} / \mathrm{m}^{2}\right)$

B

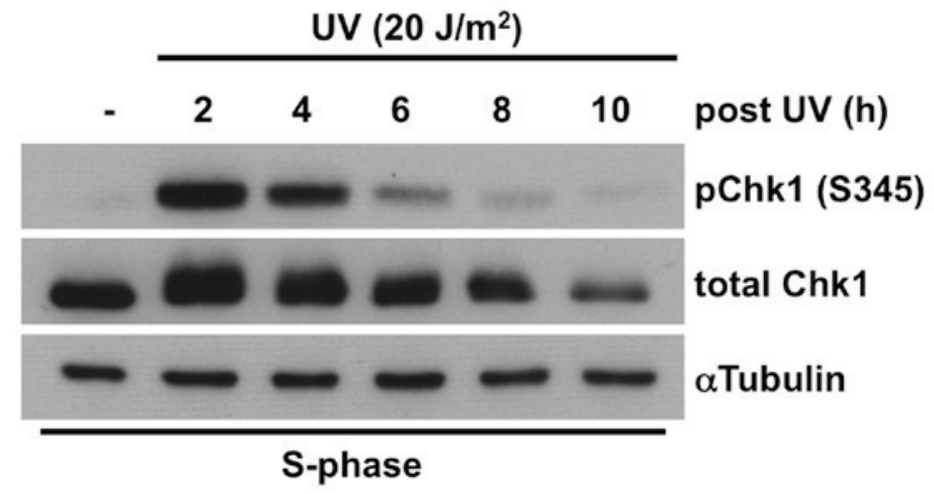

Figure 3. UV-induced CTF18-RPA interaction and Chk1 phosphorylation gradually decrease with time. (A) The dissociation kinetics of the CTF18-RPA complex in HEK293 cells. PLAs were performed with the indicated time course (magnification, x240). The Duolink Image Tool was used to quantify PLA signals. The vertical axis shows the total number of nuclear PLA signals divided from the nuclei and was normalized to non-irradiation group, and the horizontal axis indicates the time course after UV irradiation. Error bars indicate the standard error of the mean of three different fields. (B) The phosphorylation of Chk1 decreases after UV irradiation. HEK293 cells synchronized at S phase were irradiated with UV $\left(20 \mathrm{~J} / \mathrm{m}^{2}\right)$. After the indicated times, whole-cell lysates were immunoblotted with antibodies as indicated. Red fluorescent foci indicate the proximity of the two proteins and the nuclei were stainedwith Hoeschst 33342/ UV, ultraviolet; CTF18, chromosome transmission fidelity protein 18; Chk1, checkpoint kinase 1; RPA, replication protein A; PLA, proximity ligation assay; p-, phosphorylated.

replication stress responses, it is expected that the CTF18-RPA complexes could be observed in the nucleus when replication forks are stalled during $\mathrm{S}$ phase. To test this hypothesis, the PLA assay was conducted, where two endogenous proteins are immunostained with secondary antibodies, originating from different species, conjugated to complementary oligonucleotides. In this assay, when two distinct antibodies locate in close proximity $(<40 \mathrm{~nm})$, the conjugated DNA can be amplified and detected with a fluorescent probe as foci that represent molecules of each of two interacting proteins $(23,24)$. To induce the replication checkpoint response, double-thymidine arrested HEK293 cells were released into $S$ phase and subsequently treated with HU, which causes a reversible inhibition of DNA synthesis and thus blocks the progression of replication forks (25). The DuoLink assay showed that there is no significant signal in the absence of HU, whereas HU treatment resulted in the formation of nuclear PLA foci in a dose-dependent manner (Fig. 2A). These results suggest that the S-phase checkpoint response elicited by stalled replication forks leads to the interaction between CTF18 and RPA in the nucleus. 
Interaction between CTF18 and RPA occurs after $U V$ irradiation. In addition to the $\mathrm{S}$-phase checkpoint pathway, eukaryotic cells can tolerate replication stress by bypassing DNA lesions via TLS (6). Since CTF18 has been shown to be implicated in TLD (11), it was investigated whether UV-induced DNA damage triggered CTF18-RPA interaction during S phase. Synchronized HEK293 cells at early S phase were exposed to UV irradiation at 20 or $100 \mathrm{~J} / \mathrm{m}^{2}$, and after $2 \mathrm{~h}$, the CTF18-RPA interaction was assessed by counting PLA foci. A few foci were observed in the nucleus following exposure to $20 \mathrm{~J} / \mathrm{m}^{2} \mathrm{UV}$, and the formation of nuclear foci was significantly augmented when irradiated with a high dose $\left(100 \mathrm{~J} / \mathrm{m}^{2}\right)$ of UV light compared with a low dose $\left(20 \mathrm{~J} / \mathrm{m}^{2}\right.$; Fig. 2B). These findings suggest that the CTF18-RPA interaction occurs in response to the initiation of translesion DNA synthesis, although it is impossible to exclude the possibility that the interaction may result from the S-phase checkpoint response by UV irradiation.

Kinetics of the CTF18-RPA interaction correlate positively with that of Chkl phosphorylation. Finally, it was hypothesized that if the CTF18-RPA interaction is required for the replication stress responses, it may be sustained until the attenuation of the S-phase checkpoint pathway. Therefore the dissociation kinetics of the CTF18-RPA complex were examined by tracking the time course of PLA signals and comparing it with the activation status of the ATR-Chk1 signaling pathway following UV-induced replication stress. As shown in Fig. 3A, it was demonstrated that while the number of foci peaks at $2 \mathrm{~h}$ after UV irradiation, it gradually decreases with time and almost disappears before $10 \mathrm{~h}$. In addition, this time-dependent decline in UV-induced binding of CTF18 to RPA was similar to that of Chk1 phosphorylation at Ser345 (Fig. 3B). Collectively, these data imply that the CTF18-RPA interaction is involved in the replication stress response, including translesion DNA synthesis and S-phase checkpoint pathway.

\section{Discussion}

The present data demonstrated that RPA is a novel binding partner of CTF18 in mammalian cells. It was shown that this interaction was triggered when replication stress occurred and then gradually diminished in accordance with a decrease in the phosphorylation levels of Chk1 at Ser345. Accumulating evidence has shown that the CTF18-RFC complex is critical in activation of the $\mathrm{S}$ phase checkpoint and translesion DNA synthesis by interacting with DNA polymerase $\varepsilon$ and $\eta$, respectively (10-13). However, the mechanism whereby CTF18-RFC responds to replication stress and targets the stalled replication forks remains to be determined. In this study, it was hypothesized that RPA may serve as a platform for the molecular assembly of CTF18-RFC together with DNA polymerase $\varepsilon$ and $\eta$, which in turn aids in producing an efficient response to replication stress.

The in situ PLA demonstrated that replication stress induced by $\mathrm{HU}$ treatment or UV irradiation triggers the interaction between CTF18 and RPA in the nucleus. It remains to be determined how CTF18 senses replication stress and binds preferentially to RPA on ssDNA. A possible mechanism could be the phosphorylation of the RPA2 subunit in response to replication stress. Several studies have shown that stalled replication forks cause hyperphosphorylation of RPA2 at the N-terminal region through the DNA damage response pathways involving the ATR and the DNA-dependent protein kinase $(21,26-28)$. Moreover, phosphorylation of RPA2 is known to prevent its association with the replication machinery and thus be considered as a trigger for redirecting RPA functions from DNA replication to DNA damage responses $(28,29)$. In agreement with this, RPA2 phosphorylation has been reported to enhance its interactions with the ATR and the 9-1-1 checkpoint clamp $(30,31)$. Hence, although further studies are required to address the link between RPA2 phosphorylation and CTF18-RPA interaction, the present results have provided insight into the molecular basis of the initiation of the replication stress response in mammalian cells.

Among the four clamp loader complexes, the Elg1-RFC is hypothesized to act principally as an unloader for PCNA from nascent DNA after the passage of replication forks and thereby regulate PCNA levels in chromatin (32-34). In addition, Bylund and Burgers (35) demonstrated that CTF18-RFC also unloads PCNA specifically when ssDNA is coated with RPA, and they proposed a model in which this unloading activity of CTF18-RFC may contribute to establishing sister chromatid cohesion. However, considering the present result that CTF18 binds to RPA after UV-irradiation during S phase, it is possible that CTF18-RFC may remove monoubiquitinated PCNA after replicative bypass of UV-induced CPD with Polh and subsequently reload unmodified PCNA to restart normal DNA replication. Thus, the present results provide insight into the mechanism how DNA polymerases switch during TLS.

In conclusion, the present study demonstrated that CTF18 forms a complex with RPA when replication stress is elicited by hydroxyurea treatment or UV exposure during $\mathrm{S}$ phase. The interaction kinetics between CTF18 and RPA is positively associated with the phosphorylation status of Chk1. These results suggest that RPA may be a scaffold for CTF18-RFC to be recruited to stalled replication forks and respond to replication stress.

\section{Acknowledgements}

The authors would like to thank the members of Fukamizu laboratory for their helpful discussion. This study was supported by Grants-in-Aid for Scientific Research on Priority Areas (grant no. 17054004 to Professor Akiyoshi Fukamizu), Grants-in-Aid for Young Scientists (grant no. 20780237, 22688029 to Dr Hiroaki Daitoku), and Grants-in-Aid for JSPS Fellows (grant no. 25.452 to Mr. Yuta Kaneko) from the Ministry of Education, Culture, Sports, Science, and Technology, Japan.

\section{References}

1. Branzei D and Foiani M: Regulation of DNA repair throughout the cell cycle. Nat Rev Mol Cell Biol 9: 297-308, 2008.

2. Zeman MK and Cimprich KA: Causes and consequences of replication stress. Nat Cell Biol 16: 2-9, 2014.

3. Zou L and Elledge SJ: Sensing DNA damage through ATRIP recognition of RPA-ssDNA complexes. Science 300: 1542-1548, 2003. 
4. Choi JH, Lindsey-Boltz LA, Kemp M, Mason AC, Wold MS and Sancar A: Reconstitution of RPA-covered single-stranded DNA-activated ATR-Chk1 signaling. Proc Natl Acad Sci USA 107: 13660-13665, 2010.

5. Smits VA and Gillespie DA: DNA damage control: Regulation and functions of checkpoint kinase 1. FEBS J 282: 3681-3692, 2015.

6. Yang W: An overview of Y-Family DNA polymerases and a case study of human DNA polymerase eta. Biochemistry 53: 2793-2803, 2014

7. Davies AA, Huttner D, Daigaku Y, Chen S and Ulrich HD: Activation of ubiquitin-dependent DNA damage bypass is mediated by replication protein a. Mol Cell 29: 625-636, 2008.

8. Watanabe K, Tateishi S, Kawasuji M, Tsurimoto T, Inoue H and Yamaizumi M: Rad18 guides poleta to replication stalling sites through physical interaction and PCNA monoubiquitination. EMBO J 23: 3886-3896, 2004.

9. Despras E, Daboussi F, Hyrien O, Marheineke K and Kannouche PL: ATR/Chk1 pathway is essential for resumption of DNA synthesis and cell survival in UV-irradiated XP variant cells. Hum Mol Genet 19: 1690-1701, 2010.

10. García-Rodríguez LJ, De Piccoli G, Marchesi V, Jones RC, Edmondson RD and Labib K: A conserved Pole binding module in $\mathrm{Ctf} 18-\mathrm{RFC}$ is required for $\mathrm{S}$-phase checkpoint activation downstream of Mec1. Nucleic Acids Res 43: 8830-8838, 2015.

11. Shiomi Y, Masutani C, Hanaoka F, Kimura $\mathrm{H}$ and Tsurimoto $\mathrm{T}$ : A second proliferating cell nuclear antigen loader complex, Ctf18-replication factor C, stimulates DNA polymerase eta activity. J Biol Chem 282: 20906-20914, 2007.

12. Crabbé L, Thomas A, Pantesco V, De Vos J, Pasero P and Lengronne A: Analysis of replication profiles reveals key role of RFC-Ctf18 in yeast replication stress response. Nat Struct Mol Biol 17: 1391-1397, 2010.

13. Kubota T, Hiraga S, Yamada K, Lamond AI and Donaldson AD: Quantitative proteomic analysis of chromatin reveals that Ctf 18 acts in the DNA replication checkpoint. Mol Cell Proteomics 10: M110, 2011.

14. Mailand N, Gibbs-Seymour I and Bekker-Jensen S: Regulation of PCNA-protein interactions for genome stability. Nat Rev Mol Cell Biol 14: 269-282, 2013.

15. Kanellis P, Agyei R and Durocher D: Elg1 forms an alternative PCNA-interacting RFC complex required to maintain genome stability. Curr Biol 13: 1583-1595, 2003.

16. Bellaoui M, Chang M, Ou J, Xu H, Boone C and Brown GW: Elg1 forms an alternative RFC complex important for DNA replication and genome intergrity. EMBO J 22: 4304-4313, 2003

17. Parrilla-Castellar ER, Arlander SJ and Karnitz L: Dial 9-1-1 for DNA damage: The Rad9-Hus1-Rad1 (9-1-1) clamp complex. DNA Repair (Amst) 3: 1009-1014, 2004.

18. Hanna JS, Kroll ES, Lundblad V and Spencer FA: Saccharomyces cerevisiae CTF18 and CTF4 are required for sister chromatid cohesion. Mol Cell Biol 21: 3144-3158, 2001.

19. Taniguchi T, Garcia-Higuera I, Andreassen PR, Gregory RC, Grompe M and D'Andrea AD: S-phase-specific interaction of the Fanconi anemia protein, FANCD2, with BRCA1 and RAD51. Blood 100: 2414-2420, 2002
20. Yamagata K, Daitoku H, Takahashi Y, Namiki K, Hisatake K, Kako K, Mukai H, Kasuya Y and Fukamizu A: Arginine methylation of FOXO transcription factors inhibits their phosphorylation by Akt. Mol Cell 32: 221-231, 2008.

21. Vassin VM, Anantha RW, Sokolova E, Kanner S and Borowiec JA Human RPA phosphorylation by ATR stimulates DNA synthesis and prevents ssDNA accumulation during DNA-replication stress. J Cell Sci 122: 4070-4080, 2009

22. Fan J and Pavletich NP: Structure and conformational change of a replication protein A heterotrimer bound to ssDNA. Genes Dev 26: 2337-2347, 2012

23. Söderberg O, Gullberg M, Jarvius M, Ridderstråle K, Leuchowius KJ, Jarvius J, Wester K, Hydbring P, Bahram F, Larsson LG and Landegren U: Direct observation of individual endogenous protein complexes in situ by proximity ligation. Nat Methods 3: 995-1000, 2006.

24. Gullberg M and Andersson AC: Visualization and quantification of protein-protein interactions in cells and tissues. Nat Methods 7: 2010

25. Mazouzi A, Velimezi G and Loizou JI: DNA replication stress: Causes, resolution and disease. Exp Cell Res 329: 85-93, 2014.

26. Liaw H, Lee D and Myung K: DNA-PK-dependent RPA2 hyperphosphorylation facilitates DNA repair and suppresses sister chromatid exchange. PLoS One 6: e21424, 2011.

27. Liu S, Opiyo SO, Manthey K, Glanzer JG, Ashley AK, Amerin C, Troksa K, Shrivastav M, Nicholoff JA and Oakley GG: Distinct roles for DNA-PK, ATM and ATR in RPA phosphorylation and checkpoint activation in response to replication stress. Nucleic Acids Res 40: 10780-10794, 2012.

28. Olson E, Nievera CJ, Klimovich V, Fanning E and Wu X: RPA2 is a direct downstream target for ATR to regulate the S-phase checkpoint. J Biol Chem 281: 39517-39533, 2006.

29. Maréchal A and Zou L: RPA-coated single-stranded DNA as a platform for post-translational modifications in the DNA damage response. Cell Res 25: 9-23, 2015.

30. Vassin VM, Wold MS and Borowiec JA: Replication protein A (RPA) phosphorylation prevents RPA association with replication centers. Mol Cell Biol 24: 1930-1943, 2004.

31. Wu X, Shell SM and Zou Y: Interaction and colocalization of Rad9/Rad1/Hus1 checkpoint complex with replication protein A in human cells. Oncogene 24: 4728-4735, 2005.

32. Wu X, Yang Z, Liu Y and Zou Y: Preferential localization of hyperphosphorylated replication protein A to double-strand break repair and checkpoint complexes upon DNA damage. Biochem J 391: 473-480, 2005.

33. Kubota T, Nishimura K, Kanemaki MT and Donaldson AD: The Elg1 replication factor C-like complex functions in PCNA unloading during DNA replication. Mol Cell 50: 273-280, 2013.

34. Lee KY, Fu H, Aladjem MI and Myung K: ATAD5 regulates the lifespan of DNA replication factories by modulating PCNA level on the chromatin. J Cell Biol 200: 31-44, 2013.

35. Bylund GO and Burgers PM: Replication protein A-directed unloading of PCNA by the Ctf18 cohesion establishment complex. Mol Cell Biol 25: 5445-5455, 2005. 